\title{
Benefits and barriers of clown care: A qualitative phenomenographical study of parents with children in clown care services
}

\author{
Amil Kusain Tan Jr, \\ Eija Metsälä, \\ Leena Hannula
}

Faculty of Health Care and Nursing, Helsinki Metropolia University of Applied Sciences, Finland

\begin{abstract}
Clowning is a form of humour. It is an art form that invites play, interaction, and laughter. Clown Care is a programme in hospitals and medical centres involving visits from specially trained hospital clowns. The aim of this paper is to describe the benefits and barriers associated with the clown care programme. A qualitative phenomenographical study was performed using a semi-structured in-depth interview of twelve parents with children receiving clown care in various hospital wards in a Finnish University Hospital for children. Clown care creates a positive emotional state, promotes interaction between parents and child, and fosters affirmative environmental conditions. Barriers include psychological and emotional state, severity of medical condition, developmental level, and timing and context. The findings suggest that clown care integration in the care for family and child is useful in the promotion of emotional and psychosocial well-being.
\end{abstract}

Keywords: child health; family; family-centred care; paediatrics; qualitative approaches.

\section{Introduction}

Clown care is a programme in hospitals and medical centres involving visits from specially trained clowns. Clown Doctors or Hospital Clowns are professional artists who undergo a rigorous training programme before working in the hospital to bring play, humour, and laughter into the facility for the benefit of the patient, family members, and staff. They come from a variety of backgrounds such as clowning, acting, physical theatre, mime, music, and 
close-up magic (Spitzer 2006). They usually work in pairs to encourage creative performance to free the child from the pressure to participate, and to offer professional and emotional support (Koller \& Gryski 2007: 17).

Professional clown doctors began working in hospitals in 1986 under a programme called the Big Apple Circus Clown Care Unit, which was started by Michael Christensen in New York City. This was the first established structured hospital clown programme. Clown Care has been operating for around twenty-six years in various hospital and residential care units such as paediatric, geriatric, palliative, end-of-life care and rehabilitation facilities. In Finland, Sairaalaklovnit ry is the recognised clown care organisation. It was founded by Lilli Sukula Lindblom in 2011. The role of clown doctors, according to Spitzer (2006), is to attend to the specific psychosocial needs and interaction of the patients. The term clown round is used to portray a clinical visit of professional hospital clowns. Ford et al. (2013) suggest that the impact of clown rounds is beyond the immediate interaction.

Oppenheim et al. (1997: 1838) have outlined several purposes of clowning specifically for children such as: helping children to find their place within the department; helping the child to master space; helping children to travel along their own path; helping children to cope with sounds and silence; helping children to preserve intimacy; helping children to cope with their bodies; helping children to move between medical theories and their own fantasies; helping children to cope with emotions; helping children to transform the department into a scene where imagination has free realm; and helping other carers. Mansson et al. (2013: 25) suggested that clowning helps children focus on something other than illness. Olsson et al. (2002: 21) and Spitzer (2006) suggested that clown care can create a warm climate, promote good interpersonal relationships, and relieve feelings of frustration, anxiety, or hostility. These emotional experiences can create boundary-transcending opportunities (Linge 2012: 1862). Furthermore, Åstedt-Kurki \& Liukkonen (1994: 183-188), Mallet (1995: 172) and Barkmann et al. (2013: 13) suggest that it can increase morale, self-esteem, and well-being. This has been reported as one of the benefits of clown care, especially for depressed people with a negative conception of themselves. In recent studies, Vagnoli et al. (2010: 937), Barkmann et al. (2013: 13) and Wolyniez et al. (2013: 52) showed that clowning can reduce stress, pain and anxiety during a painful procedure or hospitalisation. Therefore, there is a need for nurses, particularly paediatric nurses, who are in constant interaction with the patient and the family, to recognise the positive value and impact of clowning as an intervention.

\section{Purpose, aims and objectives}

The purpose of the study is to contribute to the growing body of knowledge of humour and clowning as an intervention. The aim is to explore the experience of parents with children in clown care services in the hospital. Moreover, the study aims to specifically describe parents' view of benefits and barriers of clown care to parents and children.

\section{Methodology}

According to Åkerlind (2005: 321-334), phenomenography investigates the qualitatively different ways in which people experience something or think about something. Phenomenography differs from phenomenology. Phenomenology is focused primarily on the significance of the phenomenon, while phenomenography focuses on experiences and 
subsequent perceptions of the phenomenon (Burns \& Grove 2009). In this study, the parents' experiences with children in clown care services are the main interest. The use of a qualitative approach is also about understanding the participant's perspective.

\subsection{Research setting}

The research was conducted at $\mathrm{HUCH}$ Children's Hospital in Helsinki, Finland, and was completed in 2014.

\subsection{Participants and sampling}

A purposive sampling was done to meet the inclusion and exclusion criteria. The parents were the main respondents of the study. The parents included in the study had children admitted in various wards of the hospital, spoke English, and had a previous contact with the hospital clowns of at least two sessions. Families who did not meet the inclusion criteria and those who were in a situational crisis physically, emotionally and psychologically were not included in the study. Using a purposive approach, the researcher recruited fourteen parents to participate in the study. Four refused to participate and ten were able to complete the interview. A preliminary analysis of the data from the initial group was performed to assess the redundancy and point of theoretical saturation. To ensure that theoretical saturation was achieved, two more parents were recruited. Thus, a total of twelve parents were recruited and interviewed.

\subsection{Data collection and analysis}

The researcher together with the head nurse of the unit was responsible for recruiting the respondents. Participants were given an information package and a consent form prior to the interview appointment. An in-depth interview using a semi-structured guide was used primarily to obtain data. In-depth interview is optimal for collecting data on an individual's perspectives and experiences (Burns \& Grove 2009). The interview was audio-recorded and the recording was then transcribed. During the transcription phase, the researcher prepared all the data by including all the questions of the interviewer, and all verbalisations were transcribed literally. The data was analysed using conventional content analysis. Preliminary readings were done to immerse and achieve an overview of the data as a whole and to identify units of analysis. Identified meaning units were then formed into condensed meaning units, which in turn were coded. Three expert panels conducted a preliminary inspection of the codes, and agreement was achieved through consensus. The codes were then formed into subcategories, categories and finally a global theme. Re-inspection was performed to ensure global themes were consistent with the codes.

\subsection{Ethical considerations}

Ethical considerations were addressed by seeking the ethical approval of the institutional board, giving the participants an information package and an informed consent form, getting 
the hospital's research permit and arranging a courtesy meeting with the clown care organisation informing about the study.

\section{Results}

\subsection{On benefits of clown care}

Table 1 shows the categories and subcategories that emerged from the theme of benefits of clown care as perceived by the parents.

Table 1.Theme, categories, and subcategories on benefits of clown care

\begin{tabular}{|c|c|c|c|}
\hline Themes & \multicolumn{3}{|c|}{ Benefits of clown care } \\
\hline \multirow{3}{*}{ Categories } & $\begin{array}{c}\text { I) Creates positive } \\
\text { emotional state }\end{array}$ & $\begin{array}{c}\text { II) Promotes interaction } \\
\text { between parents and } \\
\text { child }\end{array}$ & $\begin{array}{c}\text { III) Fosters affirmative } \\
\text { environmental condition }\end{array}$ \\
\hline \multirow{5}{*}{ Subcategories } & $\begin{array}{c}\text { Provides joy and } \\
\text { meaningfulness in life }\end{array}$ & $\begin{array}{c}\text { Promotes teaching and } \\
\text { learning experience }\end{array}$ & $\begin{array}{c}\text { Creates pass time and } \\
\text { break time }\end{array}$ \\
\cline { 2 - 5 } & $\begin{array}{c}\text { Provides happiness, } \\
\text { laughter and amusement }\end{array}$ & $\begin{array}{c}\text { Promotes good } \\
\text { relationship }\end{array}$ & $\begin{array}{c}\text { Creates cheerful and } \\
\text { relaxing atmosphere }\end{array}$ \\
\cline { 2 - 5 } & Enhances self-esteem & Promotes & Transforms hospital \\
\cline { 2 - 5 } & image \\
\hline
\end{tabular}

Creates positive emotional state. Participants felt that clown care provides joy, happiness, colour in life, good spirit, laughter, amusement and a sense of meaningfulness in life during their hospital stay. Furthermore, the participants noted that clown care boosts the self-esteem of the parents by providing a positive image and confidence in taking care of their sick child. It helps uplift mood, and relieves fatigue and stress. Parents agreed that clown care intervention is not only beneficial for the child but also essential to the family's well-being.

This makes it worth being here in the hospital in different positive ways... it brings joy to your life. [TN: 001]

I think that the most important thing I feel about this clown care programme is in general it brings some joy and positivity to the hospital. [TN: 012]

Promotes interaction between parents and child. Clown care is beneficial in promoting interaction between the parents and the child. Clown care according to participants promotes a teaching and/or learning experience. Respondents believed that when they see hospital clowns, not only are they entertained but also learn to come up with ideas and techniques that are useful in entertaining their own child when hospital clowns are not around. Furthermore, interaction with the parents helps improve the improvisation because hospital clowns get information directly from the parents about the preferences of the child. Some of the participants, on the other hand, felt that it promotes bonding moments between the parent and the child, and among the parents. This enhances communication and, thereby, promotes a good family relationship. 
We talked much with my daughter when they had visited us. What they did, what they said, what was funny, when she was happy, when I was happy. She asked me "Why did you (mother) laugh? When the clown was singing to you but the clown was singing to me like an opera". And then he stared at me. I couldn't hold myself back but laughed. And my daughter asked "Why did you laugh?" and I answered: "Because his eyes, they were so curious". [TN: 006]

Fosters affirmative environmental conditions. Clown care fosters affirmative environmental conditions. It creates a break during long periods of hospitalisation, makes time pass easily, creates a relaxed and cheerful atmosphere and, most importantly, transforms the image of the hospital as a frightening place for children.

We get a little break from thinking about sickness and this hospital life. It is a little break for us (parents). It feels good. [TN: 010]

Staying here (at the hospital) is monotonous. I guess, of days blend together and there's the same routine every day. So any kind of break in that routine I think it is pretty much always welcome whether it is a pizza day or the clowns coming or whatever. [TN: 013]

It has helped relax the atmosphere when something frightening comes or is happening to the child. [TN:012]

\subsection{On barriers to clown care}

Table 2 shows the categories and subcategories that emerged from the theme of barriers of clown care as perceived by the parents.

Table 2.Theme, categories, subcategories barriers identified to clown care

\begin{tabular}{|c|c|c|c|c|}
\hline Theme & \multicolumn{3}{|c|}{ Barriers to clown care } \\
\hline Categories & $\begin{array}{c}\text { I) Psychological \& } \\
\text { emotional state }\end{array}$ & $\begin{array}{c}\text { II) Severity of } \\
\text { medical condition }\end{array}$ & $\begin{array}{c}\text { III) Developmental } \\
\text { level }\end{array}$ & $\begin{array}{c}\text { IV) Timing \& } \\
\text { context }\end{array}$ \\
\hline \multirow{2}{*}{ Subcategories } & Fears and anxiety & $\begin{array}{c}\text { Heavily medicated } \\
\text { child }\end{array}$ & Receptiveness and \\
fatigue & $\begin{array}{c}\text { Severely ill } \\
\text { condition }\end{array}$ & $\begin{array}{c}\text { Developmental } \\
\text { aspect }\end{array}$ & $\begin{array}{c}\text { Timing \& } \\
\text { context }\end{array}$ \\
\hline
\end{tabular}

Psychological and emotional state. The psychological and emotional condition of the parents and the child is perceived to be a barrier to clown care if it involves e.g. fears, anxiety, pain and lack of receptiveness. Fear and anxiety emanate mostly during the initial phase of contact with the hospital clowns when the child is unfamiliar with the hospital clowns. This may lead to the child refusing or withdrawing from the clown care intervention. Various pain levels and fatigue experienced by children were reported to be barriers to clown care. Children with less severe pain and fatigue are more likely to engage in clown care. Moreover, openness, receptiveness, and involvement were crucial factors in determining the effectiveness of the clown care on the parents and children. When the parents and the child are not open to clown care, or do not recognise the possible health benefit of these services, they are likely to refuse the services. 
It can fail when the child is afraid of the clowns at the beginning. [TN: 001]

She (child) doesn't like the clowns. I think because she is afraid that hospital clowns are real doctors. She connected pain to anything in the hospital, even the clown. She stayed in the hospital since she was a baby and there are so much bad memories about illness and pain. Perhaps this experience has made her dislike the hospital clowns. I sometimes wish she would like them. But you can't force her when she says "No" and turns her face away. [TN: 003]

Severity of medical condition. If the child is heavily medicated, e.g. in the immediate postoperative period, or in severe illness, these are instances that are perceived to be barriers to effective clown care according to participants.

When the child is on like heavy medications or something like that it could be a barrier. [TN: 007]

There was an instance when she received an injection in her ankle. She was sleeping in the post anaesthesia recovery room and then the clowns came. I think when she saw it first, she was a little bit sleepy and drowsy and there was not much interaction with the hospital clowns. [TN: 012]

Developmental level. Participants believed that the developmental level and age of the child can become barriers to clown care, for example, when improvisation is not tailored to the child's developmental level and needs. Some respondents described the incongruence of improvisation, for example, when an infant child was told a satirical joke, and a fourteenyear-old had bubble blowing sessions. Furthermore, some of the participants believed that clowning was not appropriate for younger children and could only be appreciated by older children who can understand it better.

Like at 6 months old they don't know what the clown is. They don't care about them at all. Or they are just scared of them... Our daughter is so young... I think it is more important with the older children... And for older people, I mean older children I guess it is very good... Finally, I think they are good for the children if they are old enough. [TN: 004]

Our child is a little young, but getting there. It is difficult to say but probably from 2 years and up to, I do not know 7, 8, 10 years something like that. I think the kids love it. [TN: 012]

Timing and context. Timing and situation were perceived by some participants to be essential to the success of the clowning process not only for the child but for the parents as well.

I think it depends on what kind of a situation we have here in the hospital with the child. If things are going wrong, it is not so much likely that they come here and be very happy... we have had these moments that haven't worked; that they have come at the wrong time. For example when the nurse is taking blood, and then here is the child, crying out loud and the moment become chaotic. Two clowns come here, one is playing the guitar while the other one tries to stop the clown that plays the guitar without asking if it is alright that they come or should they come later on... yeah it does not work. That was very bad... And they didn't realise it that nobody even looked at them and I was just watching here and I am angry [TN: 004]

To recapitulate, benefits of clown care include creating a positive emotional state, promoting interaction between the parents and the child, and fostering affirmative environmental condition. Barriers associated with clown care include psychological and emotional state, severity of the medical conditions, developmental level and timing and context. A comparison and contrast with the available literature will be discussed in the next section. 


\section{Discussion}

Joy, happiness, laughter, amusement and a sense of meaningfulness in life are associated with positive emotional state according to the respondents. These findings are consistent with the reports of Åstedt-Kurki \& Liukkonen (1994: 183-188), Mallet (1995: 172), Olsson et al. (2002), Spitzer (2006), Linge (2012: 1862) and Barkmann et al. (2013: 13) that suggest a positive effect of clown care on psychological and emotional well-being of the children and the family. These positive effects in turn foster a good relationship between the parent and the child by enhancing communication skills and promoting interaction to create a special space where parent and child can bond. Mallet (1995: 172) has found that humour in the form of clowning can promote both verbal and non-verbal communication. For some children communication is a means, a mechanism, for coping with stress associated with hospital stay (Linge 2012: 1862). Furthermore, Sheldon (1996: 1175) and Dean \& Major (2008: 1088) state that humour fosters relationships, togetherness and closeness, and maintains human connections. McCreaddie \& Wiggins (2008: 584) believe that a therapeutic environment is essential for caring for the sick child. Therefore, having a clown care programme in the hospital can help transform environmental conditions. Clown care creates a break for the parent and child during long periods of hospitalisation. Some of the parents claim that staying in the hospital in a long-term basis is monotonous, and having the clowns in their room bring colour in their daily routine. Olsson et al. (2002: 21) and Spitzer (2006) acknowledge the benefit that clown care can have in shifting melancholy, boredom, and a routine atmosphere to a cheerful and relaxed atmosphere. Finally, Linge (2012: 1862) supports the findings that clown care transforms the image of hospital as a frightening place for the children to stay. In her work about the experience of magical relations with hospital clowns, she reported that the clowns were perceived by children as a magical safe area in between fantasy and reality.

Barriers to clown care include the patients' psychological and emotional state, severity of medical condition, developmental level, and timing and context. Studies of Åstedt-Kurki \& Liukkonen (1994: 183-188) proposed psychological benefits of humour which include the relief of anxiety, tensions, hatred, fears and uncertainty. Furthermore, McDonald (2004) suggested that the reduction of stress and anxiety improves mood, self-esteem, and coping skills. However, some participants felt that fears, anxiety and pain were barriers to clown care, which is contrary to the general belief and assumption that clown care alleviates such negative emotions. But studies of Åstedt-Kurki \& Liukkonen (1994: 183-188), McDonald (2004), Martin (2006: 1) and Wolyniez et al. (2013: 52) confer that there is empirical evidence that supports the use of humour in alleviating pain. Further interventional studies are required to enlighten us about these conflicting ideas.

Moreover, receptiveness or openness, and willingness or involvement are seen as an important psychological component in the barriers associated with clown care. Respondents reported that clown care was not effective in a child who is non-participative in the process. If one is willing to participate or cooperate, one is likely to have the full benefits of clown care as an intervention. If resistance is encountered, the success rate is also decreased. Sheldon (1996: 1175) has clearly reported that the patient must be receptive and share the values and perception of the humour to benefit from clown care. Severity of medical condition such as when a child is in a severely ill condition is seen as a barrier to clown care since the likelihood to appreciate intervention diminishes. However, there is no literature that claims to support this finding. Hunt (1993) cited in McCreaddie \& Wiggins (2008: 584) has reported that 
patients in the midst of crisis must be excluded from humour interventions. However, by crisis they mean a psychological crisis, not specifically referring to the medical condition of the patient.

In the context of developmental level, most of the participants believe that clown care is only appropriate for and appreciated by older children. They are not appreciated by young children and adolescents. This belief is not supported by the literature. McGhee (1979) cited in Degabriele \& Walsh (2010: 525) suggested that there are four stages in which humour develops among children and adolescents. Interpretation and appreciation of humour varies in each developmental stage but, nonetheless, humour is present across the life span. This statement about humour development is supported by cognitive theory of development by Jean Piaget (1952) cited in Degabriele \& Walsh (2010: 525). Linge (2012: 1862) showed in her study that it was important to consider age to better understand how the children experienced the relation with the hospital clowns, how they described the magical aspects of the encounter and how they viewed the importance of clown encounters to their own wellbeing.

Situations were seen as a barrier to clown care. Some respondents reported that a situation like when a nurse is performing venipuncture to a child or when a child is crying inconsolably are situations that must be avoided by hospital clowns. McDonald (2004) states that timing, content and cultural context are important considerations that must be taken into account. However, the question of when is the right time and right situation to perform clown care is still unclear. In the example mentioned, it may be a good time for hospital clowns to intervene to distract the child from the pain associated with needle puncture. In the study of Wolyniez et al. (2013: 52), they found a positive effect of a medical clown to distract the pain during intravenous access in the paediatric emergency department. The question of when is the right time and right context requires further consideration and research.

\subsection{Credibility, confirmability and transferability}

The credibility of the findings was established via the prolonged involvement of the researcher in the setting. In addition, contact with the clown care organisation was continued for two months with the goal of familiarising with and seeking information on the clown care structures and operation in Finland. An expert panel was used to review and check the research plan, coding of the meaning units, and content analysis. Confirmability was achieved when respondents were asked to confirm the results. This was carried out by sending the results back to them with the premise that they are the experts on this topic (Leech \& Onwuegbuzie 2007: 557). Transferability was ensured by clearly defining the inclusion criteria, methodology, and sampling on the report. Furthermore, a pilot study was initially performed to practise the interview skills of the researcher and to check whether the semistructured guide questions can elicit appropriate response.

\subsection{Study limitations}

Finnish and Swedish are the two recognised official languages in Finland. Thus, the use of the English language was one of the limitations encountered in the study, since the main researcher is a foreigner and non-native speaker of Finnish or Swedish. There were about four participants who refused to participate in the interview because they were not confident in and 
comfortable with using English as the medium of communication. Furthermore, during the interview process, there were times in which participants were having problems articulating their thoughts, finding the right words, and sometimes they could find no English equivalent when translating a word from their native language. Lastly, this is a small-scale project whose findings are difficult to be generalised outside this small data set.

\section{Conclusion}

Clown care creates a positive emotional state, promotes interaction between parents and child, and fosters affirmative environmental conditions. Barriers to clown care include psychological and emotional state, severity of medical condition, developmental level, and timing and context. There is disparity in the belief that clown care as an intervention reduces pain and allays negative emotions among respondents as compared to the available literature.

The appropriateness of a situation to perform clown care is still uncertain. Clown care interpretation and appreciation of humour are individualised and dependent upon child developmental stages. Moreover, there is no conclusive evidence to support that clown care reduces stress and anxiety. There is also no evidence to suggest that a severe medical condition is a barrier to implementing clown care. The question of when is the right time and right context to use clown care as an intervention requires further consideration. The findings suggest that clown care integration in the care for family and child may be used in the promotion of emotional and psychosocial well-being.

\section{Recommendations}

Clown care as a research area is interesting and offers a wide range of research possibilities, since there is still a scarcity of information. Ideas for further research identified by this study include: research on the role of nurses in clown care; research on nurses and medical staff's perspectives to and experiences of clown care; development of instruments to assess and measure perception, experience, barriers, benefits, and enabling factors for clown care to validate the qualitative results of the studies. Furthermore, interventional studies on the effect of clown care on reducing pain, stress, and fatigue are highly recommended. All these studies can pave the way for developing evidence-based practice in clown care.

\section{References}

Åkerlind, G. (2005). 'Variation and commonality in phenomenographic research methods'. Higher Education Research and Development 24 (4), pp. 321-334.

Åstedt-Kurki, P. \& Liukkonen, A. (1994). 'Humour in nursing care'. Journal of Advanced Nursing 20 (1), pp. 183-188.

Barkmann, C., Siem, A., Wessolowski, N. \& Schulte-Markwort, M. (2013). 'Clowning as a supportive measure in paediatrics: A survey of clowns, parents and nursing staff'. BMC Paediatrics 13, pp. 166.

Burns, N. \& Grove, S. (2009). The Practice of Nursing Research: An Appraisal, Synthesis, and Generation of Evidence. St. Louis, Missouri: Saunders, Elsevier. 
Dean, R. \& Major, J. (2008). 'From critical care to comfort care: The sustaining value of humour'. Journal of Clinical Nursing 17 (8), pp. 1088-1095.

Degabriele, J. \& Walsh, I. (2010). 'Humour appreciation and comprehension in children with intellectual disability'. Journal of Intellectual Disability Research 54 (6), pp. 525-537.

Ford, K., Courtney-Pratt, H., Tesch, L. \& Johnson, C. (2013). 'More than just clowns: Clown doctor rounds and their impact for children, families and staff'. Journal of Child Health Care 18 (3), pp. 286-296.

Hunt, A. H. (1993). 'Humour as a nursing intervention'. Cancer Nursing 16 (1), 34-39.

Koller, D. \& Gryski, C. (2007). 'The life threatened child and the life enhancing clown: Towards a model of therapeutic clowning'. Evidence-Based Complementary and Alternative Medicine 5 (1), pp. 17-25.

Leech, N. \& Onwuegbuzie, A. (2007). 'An array of qualitative data analysis tools: A call for data analysis triangulation'. School Psychology Quarterly 22 (4), pp. 557-584.

Linge, L. (2012). 'Magical attachment: Children in magical relations with hospital clowns'. International Journal of Qualitative Studies on Health and Well-being 7 (1), pp. 11862.

Mallet, J. (1995). 'Humour and laughter therapy: Complementary therapies in nursing and midwifery'. British Journal of Nursing 2 (3), pp. 172-175.

Mansson, M., Elfving, R., Petersson, C., Wahl, J. \& Tunell, S. (2013). 'Use of clowns to aid recovery in hospitalised children'. Nursing Child and Young People 25 (10), pp. 26-30.

Martin, R. (2006). 'Sense of humor and physical health: Theoretical issues, recent findings, and future directions'. Humor: International Journal of Humor Research 17 (1/2), pp. 119.

McCreaddie, M. \& Wiggins, S. (2008). 'The purpose and function of humour in health, health care and nursing: A narrative review'. Journal of Advanced Nursing 61 (6), pp. 584-595.

McDonald, C. (2004). 'A chuckle a day keeps the doctor away: Therapeutic humour and laughter'. Journal of Psychosocial Nursing 42 (3), 18-25.

McGhee, P. E. (1979). Humor: Its Origin and Development. San Francisco: Freeman.

Olsson, H., Backe, H., Sorensen, S. \& Kock, M. (2002). 'The essence of humour and its effects and functions: A qualitative study'. Journal of Nursing Management 10 (1), pp. 2126.

Oppenheim, D., Simonds, C. \& Hartmann, O. (1997). 'Clowning on children's wards'. Lancet 350 (9094), pp. 1838-1840.

Piaget, J. (1952). The Origins of Intelligence in Children. New York: International Universities Press.

Sheldon, L. (1996). 'An analysis of the concept of humour and its application to one aspect of children's nursing'. Journal of Advanced Nursing 24 (6), pp. 1175-1183.

Spitzer, P. (2006). 'Hospital clowns: Modern-day court jesters at work'. Lancet 368 (1), pp. $34-35$.

Spitzer, P. (2012). Clown Doctors. Australia: The Humour Foundation Churchill Fellow Winston Memorial Trust Press.

Vagnoli, L., Caprili, S. \& Messeri, A. (2010). 'Parental presence, clowns or sedative premedication to treat preoperative anxiety in children: What could be the most promising option?'. Paediatric Anaesthesia 20 (1), pp. 937-943.

Wolyniez, I., Rimon, A., Scolnik, D., Gruber, A., Tavor, O., Haviv, E. \& Glatstein, M. (2013). 'The effect of a medical clown on pain during intravenous access in the paediatric emergency department: A randomised prospective pilot study'. Clinical Paediatrics 52 (12), pp. 1168-1172. 